\title{
Hippuristerone A, a novel polyoxygenated steroid from the gorgonian Isis hippuris
}

\author{
Jyh-Horng Sheu, ${ }^{\mathrm{a}, *}$ Shin-Pin Chen, ${ }^{\mathrm{a}}$ Ping-Jyun Sung, ${ }^{\mathrm{a}}$ Michael Y. Chiang ${ }^{\mathrm{b}}$ and \\ Chang-Feng Daic \\ a Department of Marine Resources, National Sun Yat-Sen University, Kaohsiung 804, Taiwan \\ ${ }^{\mathrm{b}}$ Department of Chemistry, National Sun Yat-Sen University, Kaohsiung 804, Taiwan \\ ${ }^{\mathrm{c}}$ Institute of Oceanography, National Taiwan University, Taipei 106, Taiwan
}

Received 27 June 2000; revised 3 August 2000; accepted 4 August 2000

\begin{abstract}
A novel $(22 R, 23 S, 24 S)$-polyoxygenated steroid, hippuristerone A (1), has been isolated from a Taiwanese gorgonian Isis hippuris. The structure of $\mathbf{1}$ was elucidated by 1D and 2D NMR and further confirmed by a single-crystal X-ray diffraction analysis. (C) 2000 Elsevier Science Ltd. All rights reserved.
\end{abstract}

Keywords: gorgonian; Isis hippuris; hippuristerone; polyoxygenated steroid.

Previous studies on the chemical constituents of the gorgonian corals of the genus Isis have led to the isolation of several interesting spiroketal steroids. ${ }^{1-4}$ In this paper, we report the isolation and structure determination of a novel $(22 R, 23 S, 24 S)$-polyoxygenated steroid, hippuristerone A (1), which contains independent substitution groups at all the side chain carbons (C20 and C22-C25).

Specimens of the gorgonians (wet wt, $3.0 \mathrm{~kg}$ ), collected from the coral reef of Green Island, Taiwan in February 1999, were immersed successively in $n$-hexane and $\mathrm{CH}_{2} \mathrm{Cl}_{2}$. The combined crude extract was separated by $\mathrm{Si}$ gel column chromatography. Hippuristerone A (1) was obtained as colorless prisms. ${ }^{5}$ The HRFABMS of 1 established a molecular formula of $\mathrm{C}_{33} \mathrm{H}_{52} \mathrm{O}_{7}$ $\left[(\mathrm{M}+\mathrm{Na})^{+} m / z\right.$ 583.3609], implying eight degrees of unsaturation. The IR spectrum of 1 showed absorption bands for hydroxyl $\left(v_{\max } 3352 \mathrm{~cm}^{-1}\right)$, ester carbonyl $\left(v_{\max } 1726 \mathrm{~cm}^{-1}\right)$, ketone carbonyl $\left(v_{\max } 1712 \mathrm{~cm}^{-1}\right)$ groups, and C-O stretching $\left(v_{\max } 1252 \mathrm{~cm}^{-1}\right)$ in the molecule of $\mathbf{1}$. Its ${ }^{13} \mathrm{C}$ NMR spectrum showed signals for carbons of one ketone $(\delta 211.8, \mathrm{~s})$, two ester carbonyls $(\delta 171.4, \mathrm{~s} ; 169.8, \mathrm{~s})$, five carbons bonded to an oxygen $(\delta 85.6, \mathrm{~s} ; 79.4, \mathrm{~s} ; 77.2, \mathrm{~d} ; 70.1, \mathrm{~d} ; 67.4$, s), two quaternary carbons $(\delta 43.1 ; 35.6)$, eight methine, eight methylene, and nine methyl carbons. In the ${ }^{1} \mathrm{H}$ NMR spectrum, two acetoxy methyl signals $(\delta 2.12,3 \mathrm{H}, \mathrm{s} ; 1.98,3 \mathrm{H}$, s) were

*Corresponding author. Tel: 886-7-5252000 ext. 5030; fax: 886-7-5255020; e-mail: sheu@mail.nsysu.edu.tw 
observed. Therefore, metabolite 1 must be a pentacyclic compound. By the assistance of 2D NMR spectra, including COSY, HMQC, and HMBC, 1 was shown to be a steroid. The protons of the hydroxy- and acetoxy-bearing methines which show signals at $\delta 4.03(1 \mathrm{H}, \mathrm{dd}, J=8.0,8.0$ $\mathrm{Hz}$ ) and $4.63(1 \mathrm{H}, \mathrm{d}, J=10.8 \mathrm{~Hz})$ were assigned to $\mathrm{H}-16$ and $\mathrm{H}-22$, respectively. The doublets at $\delta 0.90(3 \mathrm{H}, \mathrm{d}, J=8.0 \mathrm{~Hz})$ and $0.86(3 \mathrm{H}, \mathrm{d}, J=8.0 \mathrm{~Hz})$ were attributed to $\mathrm{H}_{3}-28$ and $\mathrm{H}_{3}-29$. Furthermore, the five singlets at $\delta 1.58(3 \mathrm{H}), 1.55(3 \mathrm{H}), 1.44(3 \mathrm{H}), 1.02(3 \mathrm{H})$, and $0.96(3 \mathrm{H})$ were assigned to the resonances of $\mathrm{H}_{3}-21, \mathrm{H}_{3}-26, \mathrm{H}_{3}-27, \mathrm{H}_{3}-19$, and $\mathrm{H}_{3}-18$, respectively. Based on the consideration of molecular formula, the second acetoxy group should be attached at C-25 and one more oxygen atom had to be placed between $\mathrm{C}-17$ and $\mathrm{C}-20$ to form a tetrasubstituted epoxide. The ketone functionality at C-3 was confirmed by its $\mathrm{HMBC}$ correlations to $\mathrm{H}_{2}-4$ and $\mathrm{H}_{2}$-2. Several ${ }^{1} \mathrm{H}-{ }^{1} \mathrm{H}$ COSY and $\mathrm{HMBC}$ correlations provided unambiguous evidence for the side chain (Fig. 1 and Table 1).
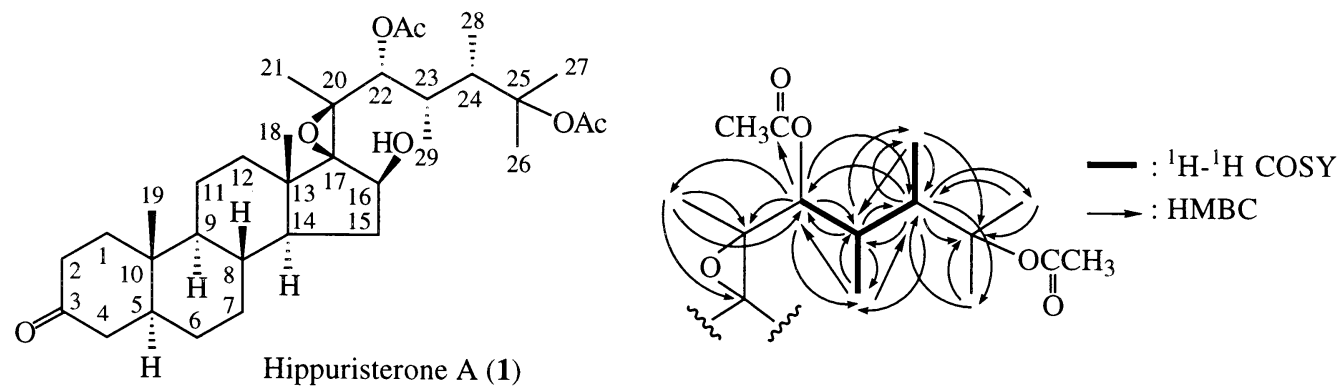

Figure 1. HMBC and ${ }^{1} \mathrm{H}-{ }^{1} \mathrm{H}$ COSY correlations of the side chain of 1

Table 1

Selective ${ }^{1} \mathrm{H}-{ }^{1} \mathrm{H}$ COSY, HMBC, and NOESY correlations for $\mathbf{1}$

\begin{tabular}{llll}
\hline $\mathrm{C} / \mathrm{H}$ & ${ }^{1} \mathrm{H}-{ }^{1} \mathrm{H}$ COSY & $\mathrm{HMBC}$ & NOESY \\
\hline 3 & & $\mathrm{H}-2 \alpha / \beta, \mathrm{H}-4 \alpha / \beta$ & \\
16 & $\mathrm{H}-15 \alpha / \beta$ & $\mathrm{H}-15 \beta$ & $\mathrm{H}-14, \mathrm{OH}-16, \mathrm{H}-22, \mathrm{H}-23, \mathrm{H}-24$ \\
17 & & $\mathrm{H}_{3}-18, \mathrm{H}_{3}-21$ & \\
20 & & $\mathrm{H}-16, \mathrm{H}_{3}-21, \mathrm{H}-22$ & \\
21 & & $\mathrm{H}-22$ & $\mathrm{H}-16, \mathrm{OH}-16, \mathrm{H}-24$ \\
22 & $\mathrm{H}-23$ & $\mathrm{H}_{3}-21, \mathrm{H}-23, \mathrm{H}-24, \mathrm{H}_{3}-29$ & $\mathrm{H}-16$ \\
23 & $\mathrm{H}-22, \mathrm{H}-24$, & $\mathrm{H}-22, \mathrm{H}-24, \mathrm{H}_{3}-28, \mathrm{H}_{3}-29$ & \\
& $\mathrm{H}-29$ & $\mathrm{H}-16, \mathrm{H}-22$ \\
24 & $\mathrm{H}-23, \mathrm{H}_{3}-28$ & $\mathrm{H}-22, \mathrm{H}-23, \mathrm{H}_{3}-26, \mathrm{H}_{3}-27, \mathrm{H}_{3}-28, \mathrm{H}_{3}-29$ & \\
25 & & $\mathrm{H}-24, \mathrm{H}_{3}-26, \mathrm{H}_{3}-27, \mathrm{H}_{3}-28$ & \\
26 & & $\mathrm{H}-24$ & \\
27 & & $\mathrm{H}-24$ & $\mathrm{H}-23, \mathrm{H}-24$ \\
28 & $\mathrm{H}-24$ & $\mathrm{H}-22, \mathrm{H}-23, \mathrm{H}-24$ & \\
29 & $\mathrm{H}-23$ & $\mathrm{H}-22$ & \\
$22-\mathrm{OCOMe}$ & &
\end{tabular}

The relative stereochemistry of $\mathbf{1}$ was deduced using a NOESY spectrum (Fig. 2 and Table 1). In the NOESY spectrum of $\mathbf{1}, \mathrm{H}_{3}-21$ did not give correlation with $\mathrm{H}_{3}-18$, and $\mathrm{H}-16$ was found to exhibit correlations with $\mathrm{H}-14, \mathrm{H}-22, \mathrm{H}-23$, and $\mathrm{H}-24$. Thus, $\mathrm{H}-16$ should be placed on the $\alpha$ phase. Detailed consideration of molecular models, suggested that compound $\mathbf{1}$ might be a 
$17 \beta, 20 \beta$-epoxy derivative and the side chain substituents of $\mathbf{1}$ should possess orientation of

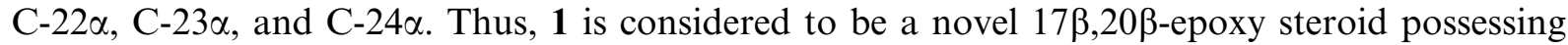
an unprecedented $(22 R, 23 S, 24 S)-23,24$-dimethyl-22,25-diacetoxyl side chain in the molecule. A single-crystal X-ray structure analysis was carried out in order to confirm the molecular

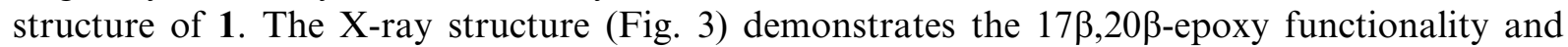
the $\beta$-orientation of $16-\mathrm{OH}$. Also, the substituents and configurations at side chain carbons $(\mathrm{C} 22-\mathrm{C} 25)$ were established unambiguously as represented in the molecular structure of $1 .^{6}$

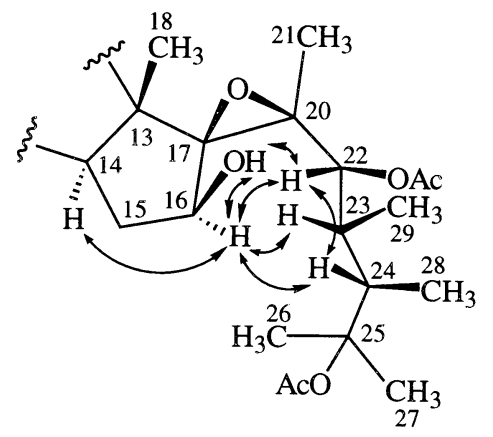

Figure 2. Selected NOE correlations of 1

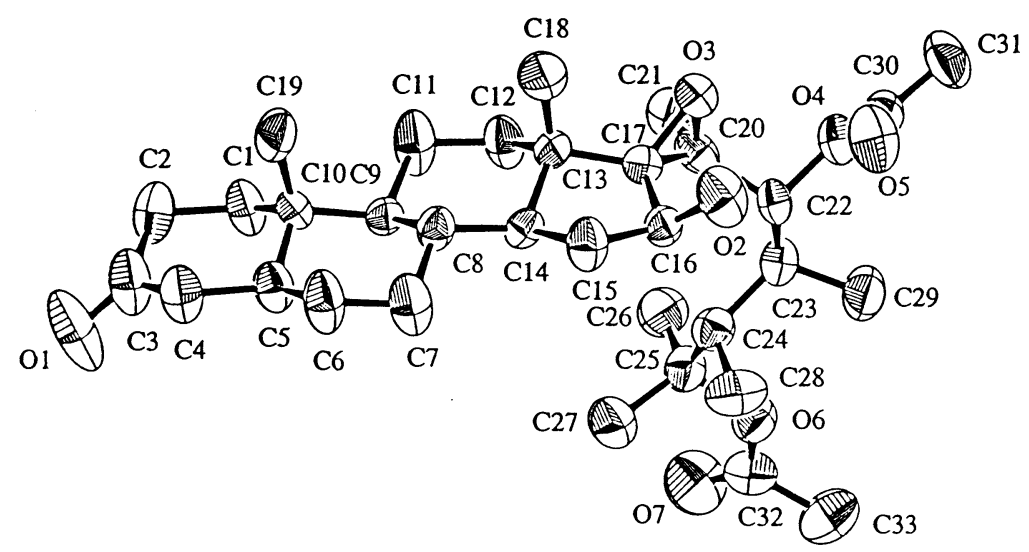

Figure 3. A computer-generated ORTEP plot of $\mathbf{1}$ showing the relative configuration. Hydrogen atoms have been omitted for clarity

\section{Acknowledgements}

This work was supported by a grant from the National Science Council of the Republic of China (NSC-89-2113-M-110-007) awarded to J.-H. Sheu.

\section{References}

1. Kazlauskas, R.; Murphy, P. T.; Quinn, R. J.; Wells, R. J.; Schönholzer, P. Tetrahedron Lett. 1977, 4439-4442.

2. Higa, T.; Tanaka, J.; Tsukitani, Y.; Kikuchi, H. Chem. Lett. 1981, 1647-1650. 
3. Higa, T.; Tanaka, J.; Tachibana, K. Tetrahedron Lett. 1981, 22, 2777-2780.

4. Rao, C. B.; Ramana, K. V.; Rao, D. V.; Fahy, E.; Faulkner, D. J. J. Nat. Prod. 1988, 51, 954-958.

5. Hippuristerone A (1) was obtained as colorless prisms $(14.2 \mathrm{mg})$, and eluted with $n$-hexane $/ \mathrm{EtOAc}=4 / 1 ; \mathrm{mp}$ $153-154^{\circ} \mathrm{C} ;[\alpha]_{\mathrm{D}}^{25}+17^{\circ}\left(c 0.5, \mathrm{CHCl}_{3}\right)$; IR $(\mathrm{KBr}) v_{\max } 3352,1726,1712$, and $1252 \mathrm{~cm}^{-1} ;{ }^{1} \mathrm{H} \mathrm{NMR}\left(\mathrm{CDCl}_{3}, 500\right.$ $\mathrm{MHz}) \delta 4.63(1 \mathrm{H}, \mathrm{d}, J=10.8 \mathrm{~Hz}, \mathrm{H}-22), 4.03(1 \mathrm{H}, \mathrm{dd}, J=8.0,8.0 \mathrm{~Hz}, \mathrm{H}-16), 3.23(1 \mathrm{H}, \mathrm{br}$ s, OH-16), 2.35 (1H, ddd, $J=15.0,10.5,7.0 \mathrm{~Hz}, \mathrm{H}-2 \beta), 2.33(1 \mathrm{H}, \mathrm{m}, \mathrm{H}-2 \alpha), 2.31(1 \mathrm{H}, \mathrm{m}, \mathrm{H}-4 \beta), 2.25(1 \mathrm{H}, \mathrm{m}, \mathrm{H}-23), 2.20(1 \mathrm{H}, \mathrm{dd}$, $J=8.0,3.5 \mathrm{~Hz}, \mathrm{H}-15 \alpha), 2.12(3 \mathrm{H}, \mathrm{s}$, acetate methyl), $2.10(1 \mathrm{H}, \mathrm{m}, \mathrm{H}-4 \alpha), 2.01(1 \mathrm{H}, \mathrm{dd}, J=5.0,1.5 \mathrm{~Hz}, \mathrm{H}-1 \alpha), 1.98$ $(3 \mathrm{H}, \mathrm{s}$, acetate methyl), $1.94(1 \mathrm{H}, \mathrm{q}, J=8.0 \mathrm{~Hz}, \mathrm{H}-24), 1.81(1 \mathrm{H}, \mathrm{m}, \mathrm{H}-12 \beta), 1.62(1 \mathrm{H}, \mathrm{m}, \mathrm{H}-11 \alpha), 1.59(1 \mathrm{H}, \mathrm{m}$, H-8), 1.58 (3H, s, $\left.\mathrm{H}_{3}-21\right), 1.55\left(3 \mathrm{H}, \mathrm{s}, \mathrm{H}_{3}-26\right), 1.53$ (1H, m, H-5), 1.47 (1H, m, H-12 2$), 1.44\left(3 \mathrm{H}, \mathrm{s}, \mathrm{H}_{3}-27\right), 1.40$

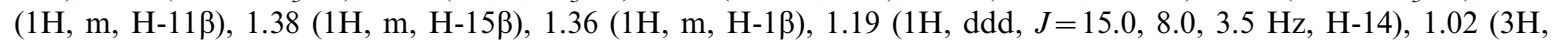
$\left.\mathrm{s}, \mathrm{H}_{3}-19\right), 0.96\left(3 \mathrm{H}, \mathrm{s}, \mathrm{H}_{3}-18\right), 0.90\left(3 \mathrm{H}, \mathrm{d}, J=8.0 \mathrm{~Hz}, \mathrm{H}_{3}-28\right), 0.86\left(3 \mathrm{H}, \mathrm{d}, J=8.0 \mathrm{~Hz}, \mathrm{H}_{3}-29\right)$, and $0.79(1 \mathrm{H}, \mathrm{dt}$, $J=8.4,2.8 \mathrm{~Hz}, \mathrm{H}-9) ;{ }^{13} \mathrm{C} \mathrm{NMR}\left(\mathrm{CDCl}_{3}, 125 \mathrm{MHz}\right) \delta 211.8$ (s, C-3), 171.4 (s, acetate carbonyl), 169.8 (s, acetate carbonyl), 85.6 (s, C-25), 79.4 (s, C-17), 77.2 (d, CH-22), 70.1 (d, CH-16), 67.4 (s, C-20), 53.2 (d, CH-9), 48.4 (d, CH-14), 46.5 (d, CH-5), 44.6 (t, $\left.\mathrm{CH}_{2}-4\right), 43.1$ (s, C-13), 40.2 (d, CH-24), 38.4 (t, $\left.\mathrm{CH}_{2}-1\right), 38.1\left(\mathrm{t}, \mathrm{CH}_{2}-2\right), 36.3(\mathrm{t}$, $\left.\mathrm{CH}_{2}-12\right), 35.6$ (s, C-10), 34.6 (d, CH-8), 33.5 (d, CH-23), $33.3\left(\mathrm{t}, \mathrm{CH}_{2}-15\right), 31.4\left(\mathrm{t}, \mathrm{CH}_{2}-7\right), 28.7\left(\mathrm{t}, \mathrm{CH}_{2}-6\right), 25.1$ (q, $\left.\mathrm{CH}_{3}-27\right), 23.0$ (q, $\left.\mathrm{CH}_{3}-26\right), 22.6$ (q, acetate methyl), 21.3 (t, $\left.\mathrm{CH}_{2}-11\right), 21.0$ (q, acetate methyl), 16.5 (q, $\left.\mathrm{CH}_{3}-21\right)$, $15.5\left(\mathrm{q}, \mathrm{CH}_{3}-18\right), 11.9\left(\mathrm{q}, \mathrm{CH}_{3}-29\right), 11.4\left(\mathrm{q}, \mathrm{CH}_{3}-19\right)$, and $10.4\left(\mathrm{q}, \mathrm{CH}_{3}-28\right)$; FABMS $m / z 583$ [2 (M+Na) $\left.{ }^{+}\right], 501$ (0.7), 483 (2), 441 (2), 423 (2), 371 (3), 341 (6), 313 (5), and 43 (100); HRFABMS 583.3609 ( $\mathrm{C}_{33} \mathrm{H}_{52} \mathrm{O}_{7} \mathrm{Na}$ calcd 583.3611).

6. Atomic coordinates for this structure have been deposited with the Cambridge Crystallographic Data Centre, 12 Union Road, Cambridge CB2 1EZ, UK. 